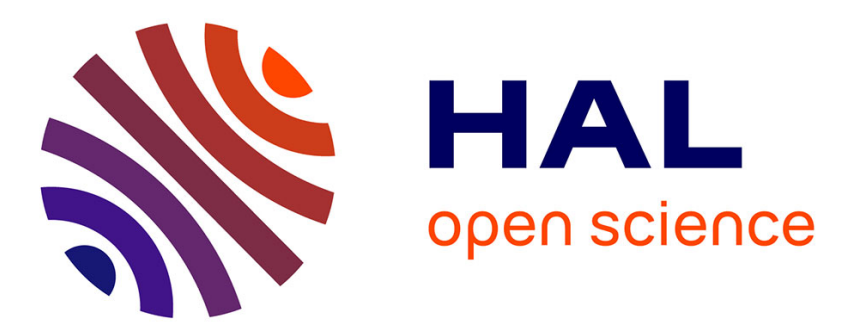

\title{
THE MODELING OF LPCVD IN SINGLE-WAFER REACTORS AS A TOOL FOR PROCESS OPTIMIZATION AND EQUIPMENT DESIGN
}

\author{
C. Kleijn
}

\section{- To cite this version:}

C. Kleijn. THE MODELING OF LPCVD IN SINGLE-WAFER REACTORS AS A TOOL FOR PROCESS OPTIMIZATION AND EQUIPMENT DESIGN. Journal de Physique IV Proceedings, 1991, 02 (C2), pp.C2-19-C2-31. 10.1051/jp4:1991202 . jpa-00249769

HAL Id: jpa-00249769

https://hal.science/jpa-00249769

Submitted on 1 Jan 1991

HAL is a multi-disciplinary open access archive for the deposit and dissemination of scientific research documents, whether they are published or not. The documents may come from teaching and research institutions in France or abroad, or from public or private research centers.
L'archive ouverte pluridisciplinaire HAL, est destinée au dépôt et à la diffusion de documents scientifiques de niveau recherche, publiés ou non, émanant des établissements d'enseignement et de recherche français ou étrangers, des laboratoires publics ou privés. 


\title{
THE MODELING OF LPCVD IN SINGLE-WAFER REACTORS AS A TOOL FOR PROCESS OPTIMIZATION AND EQUIPMENT DESIGN
}

\author{
C.R. KLEIJN \\ Delft University of Technology, Faculty of Applied Physics, Po \\ Box 5046, 2600 GA Delft, The Netherlands
}

\begin{abstract}
An overview is given of the modeling of the hydrodynamics, transport phenomena and chemical reactions in single-wafer LPCVD reactors, both at the macroscopic (reactor-scale) and at the microscopic (feature-scale) level. Examples of modeling results for single-wafer silicon LPCVD from silane and tungsten LPCVD from tungsten hexafluoride and hydrogen are presented and comparisons are made with experimental results. The use of mathematical models in the design and optimization of single-wafer LPCVD reactors and processes is discussed.
\end{abstract}

\section{INTRODUCTION}

The properties of thin films grown by CVD are highly determined by the interacting hydrodynamics, transport phenomena and chemical reactions in the CVD reactor chamber. This complex interaction obscures the basic underlying mechanisms. As a result, the development of new CVD processes and reactors, fulfilling the increasing demands in the electronics and optoelectronics industry, is a difficult and time-consuming task, which until now is mainly done by methods of trial-and-error. However, the need for mathematical CVD models, giving a detailed description of the relevant physical and chemical processes, has been recognized at an early stage. In the last two decades CVD models have evolved from simple analytical models to advanced numerical computer codes, describing the three dimensional hydrodynamics and the multi-step chemical reaction mechanisms involved [1]. Such models may be used as an aid in reactor design and process optimization, allowing a great saving in the costly and time consuming development and step-by-step improvement of reactor prototypes and process conditions. Besides, CVD models may serve as a tool for unraveling the basic physico-chemical mechanisms in CVD and in the identification and interpretation of diagnostic experiments.

Traditionally, CVD modeling has been aimed at (i) Atmospheric pressure CVD in conventional coldwall systems, such as the horizontal reactor, the barrel reactor and the pancake reactor; and (ii) Low pressure CVD in hotwall batch reactors. The modeling of coldwall atmospheric pressure reactors involves complex mixed convection flows, strong thermal diffusion phenomena and multiple reaction gas-phase chemistry. In hotwall LPCVD reactors the fluid flow is relatively simple and gas-phase chemistry is less important, but the modeling is complicated by the accurate description of heterogeneous reaction kinetics, free molecular flow phenomena and multi-component diffusion phenomena.

In the last five years it became clear that these conventional types of reactors are unsuitable for the purpose of unraveling basic CVD mechanisms. Instead, reactor geometries with a simple and well defined flow and temperature field, such as stagnation flow, impinging jet and rotating disk reactors, have been suggested for fundamental experimental and modeling studies [1-3]. In the idealized case, the hydrodynamics in these configurations can be described by means of one-dimensional transport equations [4,5]. This simplification allows for the inclusion of complex chemical mechanisms in the CVD model. Thus, detailed modeling studies have been made of various atmospheric pressure CVD processes in these idealized configurations [6-9]. In actual reactor systems, the ideal flow behavior will be 
disturbed by buoyancy driven flow recirculations, edge effects and the influence of reactor walls [10-12], necessitating a fully two-dimensional axisymmetric description of the hydrodynamics [13-17]. Moreover, symmetry-breaking effects may lead to three dimensional hydrodynamics in cylindrical symmetric reactor configurations $[17,18]$.

At the same time, with the development of new CVD processes, the increasing wafer dimensions and the ever increasing process demands in the IC industry, the interest in coldwall single-wafer LPCVD reactors has grown considerably. As a basic concept for these reactors, the main interest is again in impinging jet, stagnation flow and rotating disk configurations. Single-wafer LPCVD reactors offer several advantages over conventional hotwall batch type reactors, such as easy wafer handling, low particle contamination, excellent uniformity and efficient removal of reaction products. At present, single-wafer LPCVD reactors are widely used for tungsten deposition in ULSI metallization. Besides, there is an increasing interest in the application of single-wafer LPCVD reactors for other processes, such as in situ doped polysilicon and epitaxial silicon. However, despite its industrial relevance, the modeling of low pressure single-wafer reactors has not received as much attention as conventional low pressure and atmospheric pressure CVD processes.

As far as modeling is concerned, single-wafer LPCVD reactors combine some of the aspects of LPCVD batch reactors and atmospheric pressure coldwall systems. Mathematical models for single-wafer LPCVD should include mixed convection gas flow phenomena, multi-component ordinary and thermal diffusion, mixture composition dependent gas properties, free molecular flow phenomena and multiple homogeneous and heterogeneous chemical reactions. The modeling can take place at two geometric levels: (i) At the macroscopic, reactor-scale level, where the gas mixture can be treated as a continuum at pressures above circa $10 \mathrm{~Pa}$ and (ii) at the microscopic, feature-scale level, where the gas can no longer be treated as a continuum. The macroscopic behavior of the process is described by the combination of a hydrodynamic model for the gas flow and transport phenomena, a chemical model for the gas-phase and surface reactions and a heat transfer model for the wall and wafer temperatures. Feature-scale submodels, accounting for free molecular flow phenomena, may relate the macroscopic process conditions to microscopic process qualities.

In this paper an overview will be given of the modeling of single-wafer LPCVD processes, both at the reactor-scale and the feature-scale level and examples will be presented of its use in equipment design and process optimization. The discussion will be limited to thermal CVD processes and to process conditions for which the gas can be treated as a continuum at the reactor-scale level.

\section{HYDRODYNAMIC MODELS FOR SINGLE-WAFER LPCVD REACTORS}

Transport equations

The modeling of the reactor-scale transport phenomena (laminar gas flow, heat transfer and gas species transport) in single-wafer LPCVD reactors involves the solution of a set of coupled partial differential equations, representing the balance equations for total mass (continuity equation), momentum (Navier-Stokes equation), heat (thermal energy equation) and species (species balance equations) [19]. The cylindrical shape of most single-wafer reactors allows for a two-dimensional axisymmetric treatment of the hydrodynamics, since the occurrence of symmetry breaking effects is limited to high Rayleigh number flow conditions, which are not usually encountered under low pressure conditions [17,18]. We thus obtain for the continuity, radial momentum and axial momentum equations in axisymmetric form (A list of symbols is provided at the end of this paper):

$$
\begin{aligned}
\frac{\partial \rho}{\partial t}=-\frac{1}{\mathrm{r}} & \frac{\partial}{\partial \mathrm{r}}(\mathrm{r} \rho \mathrm{v})-\frac{\partial}{\partial z}(\rho \mathrm{u}) \\
\frac{\partial}{\partial \mathrm{t}}(\rho \mathrm{v})= & -\frac{1}{\mathrm{r}} \frac{\partial}{\partial \mathrm{r}}\left(r \rho v^{2}\right)-\frac{\partial}{\partial z}(\rho \mathrm{uv})+\frac{1}{\mathrm{r}} \frac{\partial}{\partial \mathrm{r}}\left(2 \mu \mathrm{r} \frac{\partial \mathrm{v}}{\partial \mathrm{r}}\right)+\frac{\partial}{\partial z}\left(\mu \frac{\partial \mathrm{v}}{\partial z}+\mu \frac{\partial \mathrm{u}}{\partial \mathrm{r}}\right) \\
& -\frac{2}{3} \frac{\partial}{\partial \mathrm{r}}\left(\mu\left(\frac{\partial \mathrm{v}}{\partial \mathrm{r}}+\frac{\mathrm{v}}{\mathrm{r}}+\frac{\partial \mathrm{u}}{\partial \mathrm{z}}\right)\right)-\frac{2 \mu \mathrm{v}}{\mathrm{r}^{2}}-\frac{\partial \mathrm{P}}{\partial \mathrm{r}}
\end{aligned}
$$




$$
\begin{aligned}
\frac{\partial}{\partial t}(\rho \mathrm{u})= & -\frac{1}{\mathrm{r}} \frac{\partial}{\partial \mathrm{r}}(\mathrm{r} \rho \mathrm{uv})-\frac{\partial}{\partial z}\left(\rho \mathrm{u}^{2}\right)+\frac{1}{\mathrm{r}} \frac{\partial}{\partial \mathrm{r}}\left(\mathrm{r} \mu \frac{\partial u}{\partial \mathrm{r}}+\mathrm{r} \mu \frac{\partial \mathrm{v}}{\partial z}\right)+\frac{\partial}{\partial z}\left(2 \mu \frac{\partial u}{\partial z}\right) \\
& -\frac{2}{3} \frac{\partial}{\partial z}\left(\mu\left(\frac{\partial v}{\partial r}+\frac{v}{r}+\frac{\partial u}{\partial z}\right)\right)-\frac{\partial \mathrm{P}}{\partial z}+\rho g
\end{aligned}
$$

These flow equations are coupled to the thermal energy equation and the species concentration equations through the temperature and mixture composition dependence of the fluid properties and through the buoyancy term. In the thermal energy equation, the Dufour (diffusion-thermol effect, viscous dissipation and compressibility effects may well be neglected under common LPCVD conditions. We then obtain

$$
\begin{aligned}
\rho c_{p} \frac{\partial T}{\partial t}= & -\rho c_{p} v \frac{\partial T}{\partial r}-\rho c_{p} u \frac{\partial T}{\partial z}+\frac{1}{r} \frac{\partial}{\partial r}\left(\lambda r \frac{\partial T}{\partial r}\right)+\frac{\partial}{\partial z}\left(\lambda \frac{\partial T}{\partial z}\right) \\
& +\sum_{i=1}^{N} \frac{1}{m_{i}} H_{i}\left(\frac{1}{r} \frac{\partial}{\partial r}\left(r g_{i, r}\right)+\frac{\partial}{\partial z}\left(g_{i, z}\right)-m_{i} \sum_{k=1}^{G} v_{i k}\left(\mathcal{R}_{k}^{g}-\mathcal{R}_{-k}^{g}\right)\right)
\end{aligned}
$$

The influence of buoyancy effects on the gas flow in coldwall single-wafer LPCVD reactors is illustrated in figure 1. Buoyancy may cause flow recirculations, the direction of which depends on the reactor orientation. These recirculations are suppressed at low pressures and high flowrates (i.e. low Grashof and high Reynolds numbers).

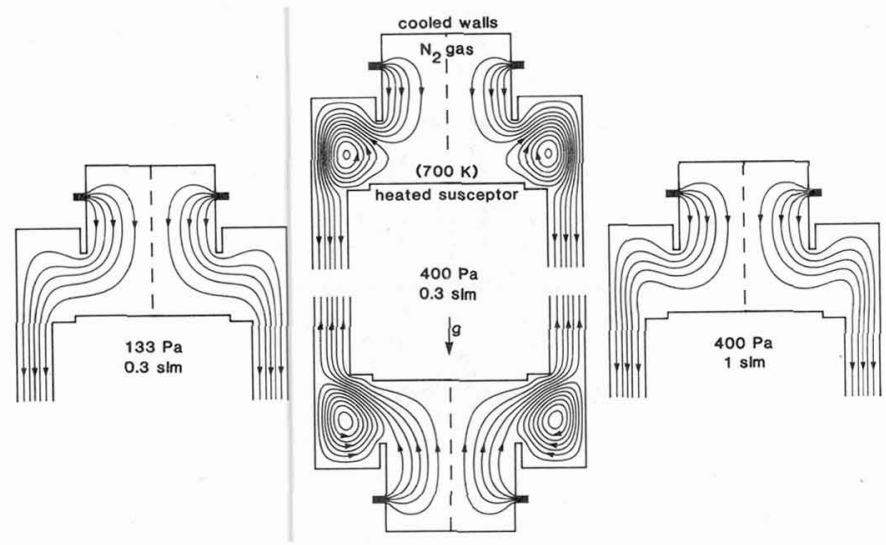

figure 1: The influence of pressure, flowrate and reactor orientation on buoyancy induced flow recirculations in a coldwall single-wafer LPCVD reactor.

The species concentrations in an $\mathrm{N}$ component gas mixture are described by $\mathrm{N}-1$ equations of the form:

$$
\frac{\partial}{\partial t}\left(\rho \omega_{i}\right)=-\frac{1}{r} \cdot \frac{\partial}{\partial r}\left(r \rho v \omega_{i}\right)-\frac{\partial}{\partial z}\left(\rho u \omega_{i}\right)-\frac{1}{r} \frac{\partial}{\partial r}\left(r g_{i, r}\right)-\frac{\partial}{\partial z}\left(g_{i, z}\right)+m_{i} \sum_{k=1}^{G} v_{i k}\left(\mathcal{R}_{k}^{g}-\mathcal{R}_{-k}^{g}\right)
$$

The homogeneous chemical reaction rates and the stoichiometric coefficients must be prescribed by a chemistry model, as will be discussed in the next section. The total species diffusion fluxes are the vector sum of ordinary diffusion fluxes and thermal diffusion fluxes. The ordinary diffusion fluxes in a multi-component mixture can be calculated from the Stefan-Maxwell equations: 


$$
\begin{aligned}
& \frac{\partial}{\partial r}\left(\omega_{i}\right)+\omega_{i} \frac{\partial(\ln M)}{\partial r}=\frac{M}{\rho} \sum_{j=1}^{N} \frac{\omega_{i} g_{j, r^{c}}^{c}-\omega_{j} g_{i, r}^{C}}{m_{j} D_{i j}} \\
& \frac{\partial}{\partial z}\left(\omega_{i}\right)+\omega_{i} \frac{\partial(\ln M)}{\partial z}=\frac{M}{\rho} \sum_{j=1}^{N} \frac{\omega_{i} g_{j, z^{c}}^{c} \omega_{j} g_{i, z}^{c}}{m_{j} D_{i j}}
\end{aligned}
$$

The thermal diffusion fluxes, which are a result of the Soret effect, causing large and heavy gas molecules to concentrate in the cold regions of the reactor, are given by

$$
\mathcal{g}_{i, r}^{T}=-\mathbb{D}_{i}^{T} \frac{\partial(\ln T)}{\partial r} ; \quad g_{i, z}^{T}=-\mathbb{D}_{i}^{T} \frac{\partial(\ln T)}{\partial z}
$$

The chemical species transport in coldwall single-wafer LPCVD reactors thus involves three physical mechanisms: convection, ordinary diffusion due to concentration gradients and thermal diffusion due to temperature gradients. This is illustrated in figure 2.

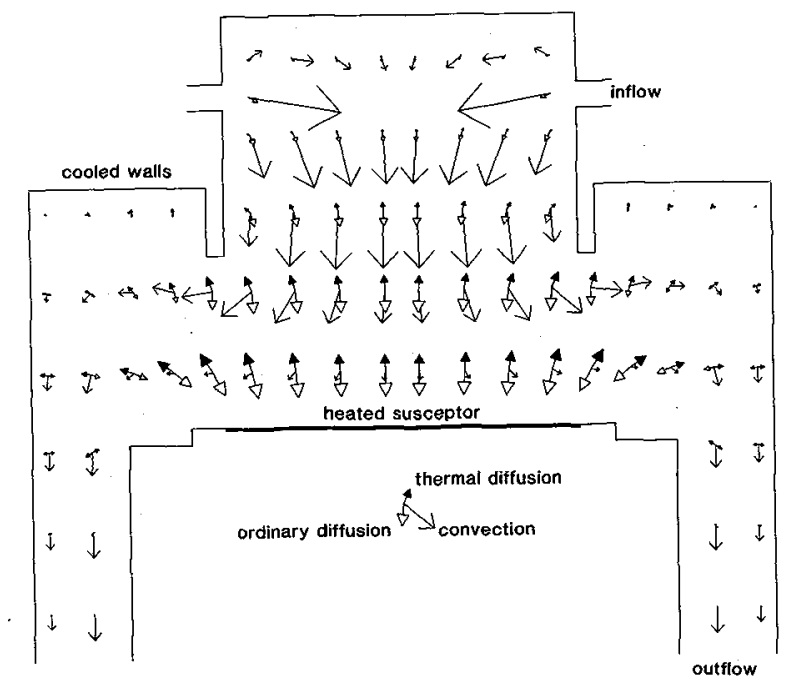

figure 2: The relative importance of the three species transport mechanisms. Shown are convective, ordinary diffusion and thermal diffusion mass fluxes of tungsten hexafluoride. (Tungsten LPCVD from $5 \mathrm{sccm}$ tungsten hexafluoride and $95 \mathrm{sccm}$ hydrogen at $133 \mathrm{~Pa}$ total pressure and $648 \mathrm{~K}$ wafer temperature).

\section{Boundary conditions}

The transport equations, which are generally applicable to any CVD reactor, must be completed with boundary conditions, defining a particular reactor configuration. These boundary conditions include prescribed velocities, temperatures and species concentrations in the inflow, zero gradients in the outflow, no-slip conditions on solid walls and zero total species fluxes normal to nonreacting walls. On reacting surfaces, the total normal flux for each species equals the net consumption rate by surface reactions and the non-zero normal velocity component is determined by the net mass deposition rate: 


$$
\begin{aligned}
& \rho \omega_{i} v_{n}+g_{i, n}^{c}+g_{i, n}^{T}=m_{i} \sum_{k=1}^{S} \sigma_{i k} \mathcal{R}_{k}^{S} \\
& v_{n}=\frac{1}{\rho} \sum_{i=1}^{N} m_{i} \sum_{k=1}^{S} \sigma_{i k} \mathcal{R}_{k}^{S}
\end{aligned}
$$

where $\mathrm{n}$ is the direction normal to the surface. The surface reaction rates and the stoichiometric coefficients are to be prescribed by a heterogeneous chemistry model, as will be discussed in the next section. For the reactor walls and the wafer surface isothermal or adiabatic thermal boundary conditions can be used. However, a more sophisticated model for the calculation of wall and wafer temperatures is often necessary. This will be discussed in section 4 .

\section{Fluid properties}

Due to the high concentrations of reactants and products, the fluid properties (density, viscosity, thermal conductivity and (thermal) diffusion coefficients) of the gas mixture in single-wafer LPCVD reactors will not only depend on the pressure and temperature, but also on the composition of the mixture. These properties may be obtained from theoretical models, as provided by Chapman-Enskogg kinetic theory of gases [20-22]. The required intermolecular force parameters of the individual gas species have been tabulated for numerous gases [23] or may be estimated from critical parameters [19]. The evaluation of fluid properties in CVD gas mixtures from kinetic theory requires a great computational effort, which may take up a significant part of the total computer power needed for numerical CVD modeling.

\section{Numerical solution methods}

Three classes of numerical methods are available for the solution of the transport equations: (i) finite difference methods, which have mainly be used for the solution of 1D problems in idealized reactor configurations [e.g. 6-8], (ii) finite element methods, which are specifically useful for modeling complex geometries [e.g. 12-13] and (iii) finite volume methods, which are easily accessible for the inclusion of complicated physical phenomena into 2D or 3D hydrodynamic models [e.g. 10,22]. Sophisticated, general purpose Computational Fluid Dynamics codes for the solution of multi-dimensional transport equations, based on finite element or finite volume methods, have become available commercially in the last decade [24]. However, these codes have not generally been developed specifically for CVD modeling. The inclusion of some CVD-specific aspects, such as multi-component (thermal) diffusion, mixture composition dependent fluid properties, multiple-step and multiplespecies homogeneous chemical reactions and heterogeneous deposition reactions, generally requires the modification of general purpose fluid dynamics codes, which may not be trivial, especially if source codes are not available [25].

\section{CHEMISTRY MODELS FOR SINGLE-WAFER LPCVD PROCESSES}

Whereas the hydrodynamic model is generally applicable to any single-wafer LPCVD process, a separate chemistry model, stating the relevant homogeneous and heterogeneous reaction pathways and rate constants, must be specified for each particular process. The lack of detailed chemistry models is still the most important bottle-neck in CVD modeling. For this reason, many CVD modelers have resorted to the use of simple chemistry models, assuming one single overall chemical reaction, with a rate expression that is obtained from empirical correlations. However, empirical correlations for a particular operating regime lose their validity when moving towards different temperatures and pressures. Moreover, intermediate reaction products may have a large influence on the deposition process. Therefore, the development of future generation CVD reactors and processes requires a more fundamental understanding of the chemistry involved. This must be obtained both from extensive experimental research and from theoretical modeling.

\section{Gas-phase chemistry modeling}

A homogeneous chemistry model should state the relevant reaction pathways and the reaction 
rates for the gas-phase chemistry. The development of a homogeneous reaction mechanism model is usually performed in two steps: First, a large system of all plausible species and elementary reactions is constructed. This system typically contains over one hundred species and reactions, prohibiting its use in CVD modeling. Therefore, in a second step, chemical kinetics sensitivity analysis and experimental gas-phase analysis studies are used to reduce the system, maintaining only the most relevant reaction pathways. The resulting mechanism should reflect the essential features of the process over a wide range of process conditions. The reaction rates of the elementary reversible reaction steps are obtained from

$$
\mathscr{R}_{\mathrm{k}}^{g}=k_{\mathrm{k}}^{g} . \prod_{\text {reactants }}\left(\frac{\mathrm{P}}{\overline{R T}} \mathrm{f}_{\mathrm{i}}\right)^{\mid v_{\mathrm{ik}} !} ; \quad \mathcal{R}_{-\mathrm{k}}^{g}=k_{-\mathrm{k}}^{g} \cdot \prod_{\text {products }}\left(\frac{\mathrm{P}}{\mathrm{RT}} \mathrm{f}_{\mathrm{i}}\right)^{\nu_{\mathrm{ik}}}
$$

If not available, the rate constants have to be determined experimentally or estimated theoretically, using statistical thermodynamics, transition state theory and bond dissociation enthalpies. Generally, the rate constants have an Arrhenius temperature dependence and are independent of the pressure at sufficiently high pressures. At low pressures however, the reactions enter their pressure fall-off regime. The classical Lindemann expression

$$
k^{g}(\mathrm{P}, \mathrm{T})=k^{g}(\mathrm{P}=\infty, \mathrm{T}) \cdot \frac{k_{1} \mathrm{P}}{k_{1} \mathrm{P}+k_{2}}
$$

provides a qualitative description for the pressure fall-of $f$ behavior of unimolecular decomposition reactions. More accurate quantitative predictions may be obtained from RiceRamsperger-Kassel-Marcus analysis [26, 27].

The development of CVD gas-phase chemistry' models has been limited to atmospheric pressure conditions mainly. Pioneering work has been performed by Coltrin and coworkers for silicon CVD from silane [8, 28-29]. More recently, models have been developed for the gasphase chemistry in the CVD of silicon dioxide from (di)silane and nitrous oxide [30] and in the MOCVD of GaAs from trimethylgallium and arsine [9,31]. In general, gas-phase chemistry is found to contribute significantly to the deposition process under atmospheric pressure CVD conditions. Its accurate modeling may involve several dozens of elementary reactions and intermediate species. As a result, the use of detailed gas-phase chemistry models has up until now been restricted to simplified iD reactor configurations.

In contrast to atmospheric pressure CVD, it is generally assumed that gas-phase reactions are negligible in LPCVD batch processes. For example, experimental observations [32] and modeling results [33] indicate, that less than $10 \%$ of the silicon deposition from silane in LPCVD batch rebctors is due intermediate species formed in the gas-phase. Likewise, model simulations and experimental gas-phase analyses indicate, that gas-phase reactions are negligible in tungsten LPCVD from tungsten hexafluoride and hydrogen or silane [34-36]. As a result, gas-phase reactions are usually neglected in LPCVD modeling. However, in singlewafer LPCVD reactors the temperature and pressure will generally be higher than in conventional batch reactors, in order to obtain a sufficiently high growth rate and throughput. As a result, gas-phase reactions may be more important. For example, model simulations of the hydrodynamics and gas-phase reactions in silicon LPCVD in a coldwall single-wafer reactor show, that gas-phase reactions may contribute significantly to the silicon growth at operating pressures which are slightly increased compared to conventional LPCVD pressures [37]. Figure 3 shows the simulated contribution of gas phase reactions to the silicon growth as a function of the total pressure. Figure 4 shows radial growth rate distributions due to direct silane adsorption and due to reactive intermediates formed in the gas phase at 1330 $\mathrm{Pa}$ total pressure. It is clear, that gas phase reactions become very important and that the growth uniformity is highly deteriorated at slightly increased pressures.

In conclusion, the accurate modeling of coldwall single-wafer LPCVD processes necessitates a careful consideration of the contribution of gas-phase reactions. For this purpose, gas-phase chemistry models will have to be (further) developed and pressure fall-off reaction rate constants will have to be determined. It is likely, that gas-phase reaction mechanisms in single-wafer LPCVD reactors are less complex than in atmospheric pressure reactors. This allows for an accurate modeling of the gas-phase chemistry in combination with a 


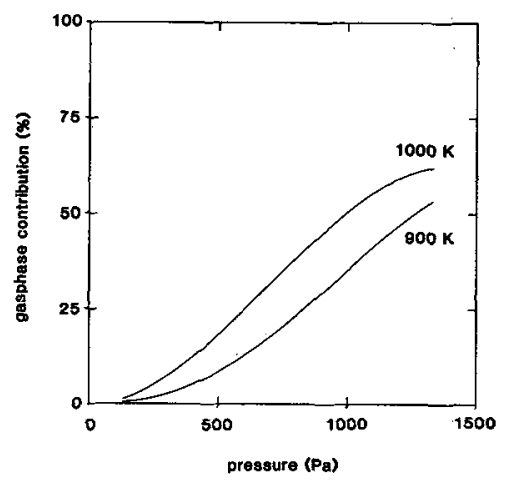

figure 3: The relative contribution of gas-phase intermediates to the silicon growth from silane (100 sccm in $900 \mathrm{sccm}$ nitrogen) in a coldwall single-wafer reactor, as a function of pressure, for two wafer tempetemperatures.

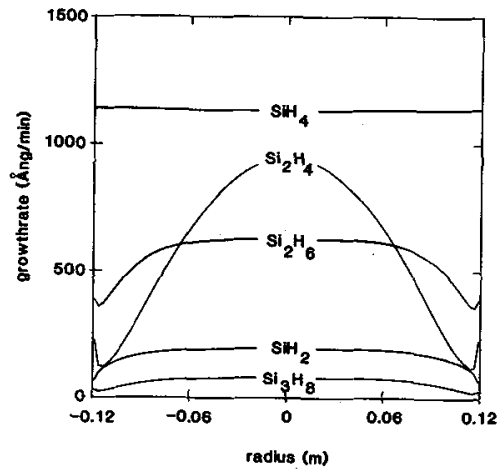

figure 4: Radial growth rate distribution on a $0.24 \mathrm{~m}$ diameter wafer, due to direct silane absorption and due to different gas-phase intermediates. (100 sccm silane in $900 \mathrm{sccm}$ nitrogen, $1000 \mathrm{~K}$ wafer temperature, $1330 \mathrm{~Pa}$ total pressure)

realistic, non-simplified description of the axisymmetric hydrodynamics in such reactors. Thus, single-wafer reactors may prove to be an excellent tool for fundamental CVD studies.

\section{Surface chemistry modeling}

It is clear, that sophisticated models for surface reaction mechanisms and kinetics are essential for the accurate modeling of single-wafer LPCVD processes. However, up until now, the fundamental modeling of heterogeneous chemical reactions in CVD has not received as much attention as gas-phase chemistry modeling. Instead, various simplifying assumptions have been used in the modeling of heterogeneous reaction mechanisms and kinetics.

The most simple approach is to assume one single overall surface reaction, with very fast kinetics compared to the maximum rate of supply of the reactants. In this case the deposition is transport limited and no detailed information is needed about the surface kinetics. Although useful for the prediction of growth rates in some atmospheric pressure epitaxial processes, this approach is not suited for single-wafer LPCVD modeling, where deposition will usually be kinetically limited. A second approach is again to assume one single overall reaction, but to use empirical relations, usually of the form

$$
\mathcal{R}^{S} \propto \exp (-\mathrm{E} / \mathrm{RT}) \cdot \mathrm{P}_{1}^{\alpha} \cdot \mathrm{P}_{2}^{\beta} \cdot \cdots
$$

as a rate expression. This approach may be useful in predicting single-wafer LPCVD growth rates for a limited range of operating conditions. However, the disadvantages of the use of overall reaction schemes and empirical rate expressions have already been mentioned before. A third way to model surface reactions is to assume that the surface reaction mechanism can be described by a series of elementary heterogeneous unimolecular decomposition reactions, the rates of which can be represented by a single reaction probability $[29,38]$. The reaction rate of each unimolecular surface reaction is then taken as the product of the number of surface collisions of the species molecules as calculated from kinetic theory, and the reaction probability:

$$
\mathcal{R}_{\mathrm{j}}^{S}=\gamma_{\mathrm{j}} \mathrm{P}_{\mathrm{j}} /\left(2 \pi \mathrm{m}_{\mathrm{j}} \mathrm{RT}\right)^{1 / 2}
$$


The reaction probability is fitted to match theory and experiment for a particular range of process conditions. This approach can only be used for unimolecular surface processes. Moreover, rather than having a fixed value, the reaction probability usually appears to be a rather complicated function of temperature and pressure [37,39]. A final approach which has been used in surface reaction modeling is to propose a particular sequence of elementary surface process steps, and to assume that one of these steps is rate limiting, whereas all the other steps are in equilibrium [40,41]. This leads to an expression for the overall reaction rate as a function of temperature and species partial pressures. The choice between different reaction schemes and rate limiting steps, leading to different rate expressions, is based on the experimental growth rate behavior of the process. The unknown constants in the rate expression are chosen to fit experimental data. This approach has for instance been used rather successfully in the modeling of the heterogeneous deposition reactions in single-wafer tungsten LPCVD from tungsten hexafluoride and hydrogen [42]. Figure 5 shows model simulations and experimental results for the dependence of the tungsten growth rate on the tungsten hexafluoride inlet pressure, at different total pressures and wafer temperatures. Figure 6 shows model simulations and experimental results for the tungsten growth rate as a function of radius on a 3 in. wafer.
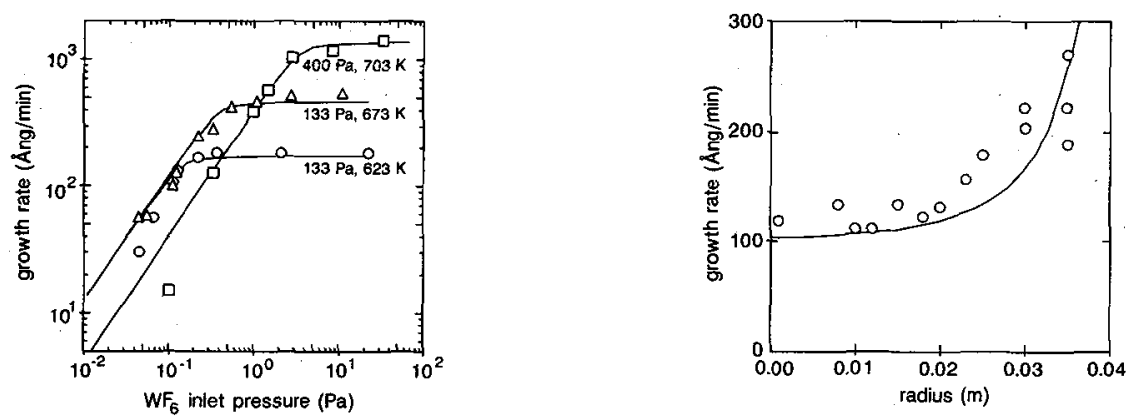

figure 5: Model simulations and experimental results for the tungsten growth rate in a coldwall single-wafer reactor as a function of the tungstien hexafluoride inlet pressure at different total pressures and wafer temperatures (after [42]). figure 6: Model simulations and experimental results for the tungsten growth rate as a function of radius on a 3 in. wafer (133 $\mathrm{Pa}$ total pressure, $673 \mathrm{~K}$ wafer temperature, $1000 \mathrm{sccm}$ hydrogen, $199 \mathrm{sccm}$ Argon, $1.3 \mathrm{sccm}$ tungstenhexafluoride). (after [42]).

Although useful in the prediction of growth rates for a limited range of process conditions, the above approaches certainly do not suffice to predict LPCVD process characteristics such as film composition and structure, doping level, and impurity incorporation. For this purpose, detailed models of the multi-step heterogeneous reaction mechanisms and kinetics, including adsorption/desorption phenomena, gas-solid reactions and surface-only reactions must be developed. Pioneering work in the detailed modeling of heterogeneous CVD processes and the $a b$ initio estimation of heterogeneous rate constants has been published by Pollard and coworkers for gallium arsenide MOCVD from trimethylgallium and arsine $[9,43]$ and for tungsten LPCVD from tungsten hexafluoride and hydrogen $[34,35]$.

\section{THE MODELING OF WAFER AND WALL TEMPERATURES}

Since the deposition processes in single-wafer LPCVD reactors are highly determined by thermally activated heterogeneous processes, it is important to have precise knowledge of the temperature of the wafer and the reactor walls. The wafer temperature distribution has a large influence on the properties, growth rate and uniformity of the deposited film. The temperature of the reactor walls may influence the gas flow pattern, gas-phase chemistry, 


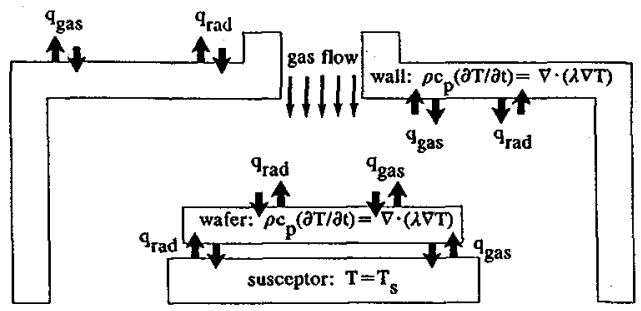

Figure 7: Heat transfer in a coldwall singlewafer reactor with susceptor heating.

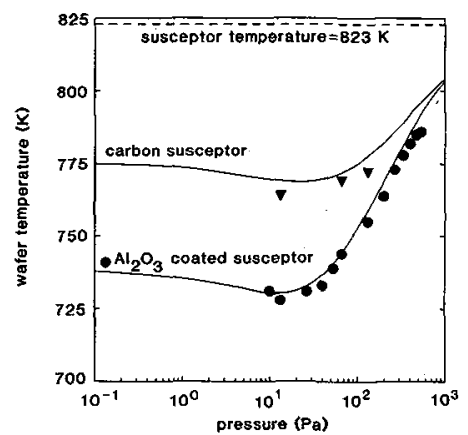

figure 8: Experimental and simulated wafer temperatures in a coldwall single-wafer reactor (after [46])

particle formation etc. The purpose of CVD reactor heat transfer models is to predict the wafer and reactor wall temperature distributions. These temperature distributions are determined by thermal conduction in the wafer and reactor walls and by radiative, conductive and convective heat fluxes between the heating source, the wafer, the gas mixture, the reactor walls and the surroundings. These heat fluxes depend on the reactor configuration, the radiative and thermal properties of the wafer, the reactor walls and the gas mixture and on the gas flow pattern in the reactor.

Figure 7 illustrates the heat transfer mechanisms in a single-wafer reactor with susceptor heating. The wall and wafer temperature distributions can be calculated by solving heat conduction equations for the wafer and the reactor wall material, with the various heat fluxes as boundary conditions. Due to surface roughnesses, the direct thermal contact between the wafer and the susceptor is minimal and heat transfer has to occur through gas particle conduction and thermal radiation. Since the mean free path length of the gas molecules is larger than or comparable to the susceptor-to-wafer distance at common LPCVD pressures, the heat conduction is governed by free molecular flow phenomena [44-46]. The calculation of the radiant heat fluxes involves the discretization of all walls into small surface elements and the determination of radiant exchange factors between each pair of surface elements. The convective heat fluxes to the reactor gases are determined by the gas flow and gas temperatures in the reactor, as calculated by the hydrodynamic model [47-49].

The modeling of wafer temperatures is of special importance in cold-wall single wafer LPCVD reactors with susceptor heating, because of the deteriorated thermal contact between susceptor and wafer at low gas pressures. Several authors have used detailed heat transfer models to study the influence of the type of carrier gas, the gas pressure and the susceptor and wafer emissivities on the temperature difference between wafer and susceptor [44-46]. It was found, that this difference may be as large as $100 \mathrm{~K}$. Figure 8 shows modeling and experimental results by Hasper [46] for the wafer temperature as a function of the total gas pressure in a cold-wall single wafer LPCVD reactor, for two types of susceptor material.

\section{FEATURE-SCALE MODELING}

Together, the hydrodynamic model, the chemistry model and the heat transfer model constitute a reactor-scale CVD model, describing the macroscopic CVD process characteristics. Besides, it may be important to predict microscopic, feature-scale process characteristics, such as the selectivity-loss in selective and the step coverage in blanket feature filling processes. In a selective process, deposition takes place on e.g. silicon and metallic surfaces, but not on e.g. oxide surfaces. This offers the possibility of filling vias and contact holes without the need for subsequent backetching. However, selectivity loss may occur, causing growth on oxide surfaces as well. Alternatively, blanket feature filling processes may be used, which should have a high conformality in order to avoid undesirable 
void formation. In an ideal blanket process, the step-coverage, i.e. the ratio of the film thickness at half the feature depth and the feature radius, is close to $100 \%$.

The feature-scale deposition characteristics depend on the local macroscopic process conditions 'at the wafer surface. However, at the feature-scale level, with typical dimensions $<1 \mu \mathrm{m}$, the gas exhibits free molecular (Knudsen flow) phenomena: The aim of featurescale CVD models is to describe these phenomena and predict their influence on microscopic film qualities. The boundary conditions for feature-scale models are determined by the local macroscopic process conditions, that may be obtained from a reactor-scale CVD model.

In this way, Ulacia and coworkers [44] used the combination of a single-wafer reactor model and a feature-scale model to predict selectivity loss in tungsten LPCVD from tungstenhexafluoride and hydrogen. McInerney et al. [50] and Hasper et al.[51,52] have used combined reactor and feature-scale models to predict the step coverage in blanket tungsten LPCVD. The step coverage model is based on $1 \mathrm{D}$ reactant balance equations, considering Knudsen diffusion in cylindrical or rectangular features and deposition on the feature walls. By comparison to SEM observations, Hasper et al. found a good agreement between model predictions and experimental step coverages in high aspect ratio trenches. Figure 9 shows modeled and experimental step coverages, as a function of a characteristic dimensionless number, the so called step coverage modulus, which involves the feature dimensions, the reactant surface concentration, the Knudsen diffusion coefficient and the growth rate [52].

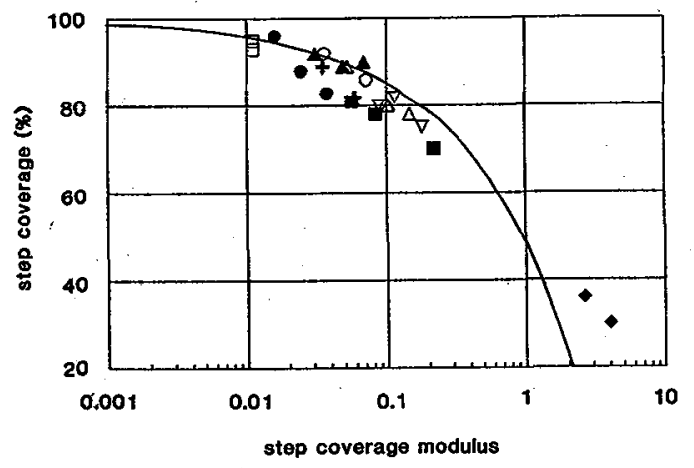

Figure 9: Experimental data and model simulations (-) of the tungsten LPCVD step coverage in high aspect ratio trenches, as a function of the dimensionless step coverage modulus (after [52]).

\section{THE USE OF SINGLE-WAFER LPCVD MODELS IN FUNDAMENTAL PROCESS RESEARCH AND AS A TOOL FOR PROCESS OPTIMIZATION AND EQUIPMENT DESIGN}

Simulation models for single-wafer LPCVD processes may be used for two main purposes:

(i) In fundamental process research, the use of simulation models may be vital in the identification and correct interpretation of diagnostic experiments. These are hampered by the fact that the process conditions at the wafer surface depend on the transport phenomena and chemical reactions in the reaction chamber and are therefore highly unknown. As a result, the validity of experimental data is of ten limited to a particular reactor configuration, making it hard to distinguish between fundamental process characteristics and reactor dependent artifacts. Simulation models, relating wafer conditions to the reactor inlet conditions, may resolve these problems. Usually, this involves some kind of iterative procedure, using model predictions to evaluate experiments, and experimental data to improve the model.

(ii) Once a reliable CVD model has been established, the model may be used to optimize reactor design and process conditions. This may involve conflicting process demands, such as uniform growth rate, high throughput, and economic use of reactants. The influence of reactor configuration and process conditions on these performance measures can be studied by means of a simulation model. Despite these great potentials, most modeling research has been limited to the description of the principal behavior of CVD processes and few have proceeded as far as to actually apply. simulations in equipment design and process optimization. 


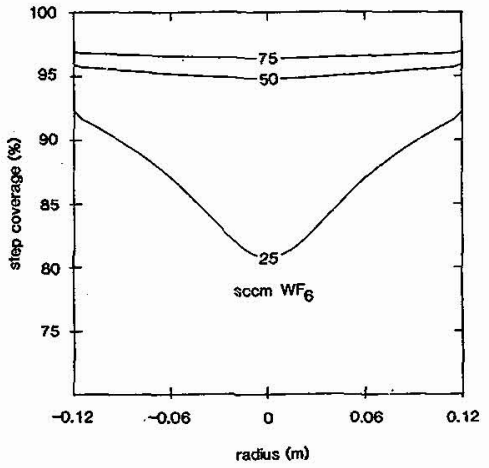

Figure 10: The influence of the tungstenhexafluoride flow on the stepcoverage in 5:1 trenches. (1064 Pa total pressure, 300 sccm total flow, $693 \mathrm{~K}$ wafer temperature).

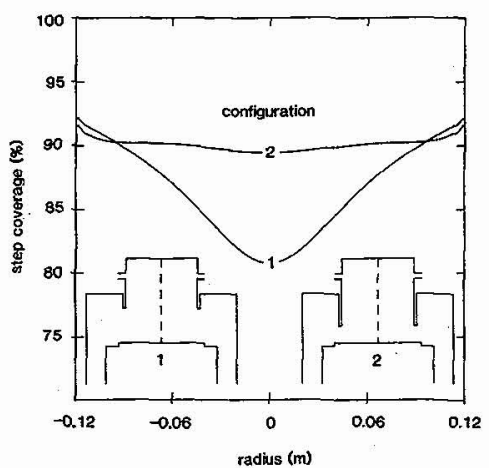

Figure 11: The influence of the reactor configuration on the step coverage in 5:1 trenches (1064 $\mathrm{Pa}$ total pressure, 200 sccm hydrogen, 25 sccm tungstenhexafluoride, $693 \mathrm{~K}$ wafer temperature)

As an example, we illustrate the optimization of a blanket tungsten feature filling process with the aid of model simulations [52]. Tungsten is deposited from tungsten hexafluoride and hydrogen in a single-wafer reactor. The process should combine a high and uniform growth rate and step coverage with a low use of expensive tungsten hexafluoride gas. With regards to multi-level metallization, the maximum temperature is limited to circa $700 \mathrm{~K}$. Model simulations, allowing easy variation of reactor geometry and process parameters, were used to optimize the process with respect to all these demands. Figures 10 and 11 show examples of the optimization of the step coverage. With the model simulations, a process has been developed which offers a high growth rate on a large wafer $(2.4 \mathrm{~nm} / \mathrm{s}$, uniform within $1 \%$ over a $0.24 \mathrm{~m}$ diameter wafer) and a high step coverage (95\% step coverage in 5:1 depth-to width aspect ratio trenches) using only $50 \mathrm{sccm}$ of tungsten hexafluoride. These model predictions were validated by experiments, showing a growth rate of $2.5 \mathrm{~nm} / \mathrm{s}$ and a step coverage > $95 \%$ in $10 \times 2 \mu \mathrm{m}$ trenches, both uniform within experimental accuracy over a 6 in. wafer. Figure 12 illustrates the extreme good step coverage obtained with this process.

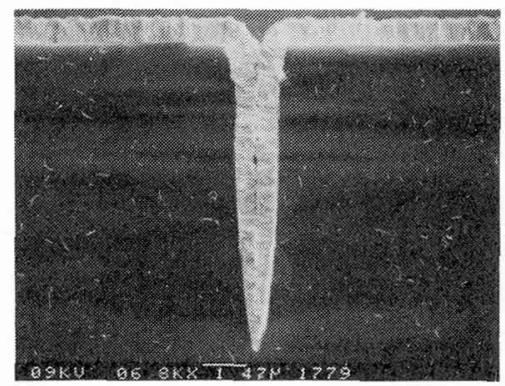

Figure 12: Step coverage in a $9 \mu \mathrm{m}$ depth $\times 1.5 \mu \mathrm{m}$ width trench, obtained with an optimized blanket tungsten feature filling process. (after [52]).

\section{CLOSING REMARKS}

Single-wafer LPCVD modeling has now come to a point were it can be used successfully in reactor design and process optimization, opening the door to the computer aided development of advanced deposition technologies for ULSI IC manufacturing. This may contribute to better process and equipment design, reduced costs, and improved IC manufacturing. However, many important challenges remain for the near future, especially with respect to the development and evaluation of detailed chemistry models. The combination of experimental and modeling research in single-wafer LPCVD reactors may be an excellent tool for this purpose. 


\section{ACKNOWLEDGEMENT}

Parts of the work described in this paper are the result of close collaboration and valuable discussions with Albert Hasper and Jisk Holleman from the University of Twente, who also performed the experiments on tungsten LPCVD growth rates and step coverage. This work forms part of the Innovative Research Program for IC Technology and was made possible by the financial support from the Netherlands Ministry of Economic Affairs.

\section{LIST OF SYMBOLS}

c specific heat, $\mathrm{J} \cdot \mathrm{kg}^{-1} \cdot \mathrm{K}^{-1}$

D binary ordinary diffusion coefficient, $\mathrm{m}^{2} \cdot \mathrm{s}^{-1}$

$\mathbb{D}^{T}$ thermal diffusion coefficient, $\mathrm{kg} \cdot \mathrm{m}^{-1} \cdot \mathrm{s}^{-1}$

$g$ gravity constant, $\mathrm{m} \cdot \mathrm{s}^{-2}$

G number of gas-phase reactions

$\mathrm{H}$ molal enthalpy, $\mathrm{J} \cdot \mathrm{mole}^{-1}$

$g$ total diffusion mass flux, $\mathrm{kg} \cdot \mathrm{m}^{-2} \cdot \mathrm{s}^{-1}$

$g^{c}$ ordinary diffusion mass flux, $\mathrm{kg} \cdot \mathrm{m}^{-2} \cdot \mathrm{s}^{-1}$

$g^{\mathrm{T}}$ thermal diffusion mass flux, $\mathrm{kg} \cdot \mathrm{m}^{-2} \cdot \mathrm{s}^{-1}$

$k^{g}$ homogeneous reaction rate constant, (in moles; $\mathrm{m}^{3}$, and $\mathrm{s}$ )

$\mathrm{m}$ mole mass, $\mathrm{kg} \cdot \mathrm{mole}^{-1}$

$\mathrm{M}$ mixture averaged mole mass, $\mathrm{kg} \cdot \mathrm{mole}^{-1}$

$\mathrm{N}$ number of gaseous species

$\mathrm{P}$ pressure, $\mathbf{P a}$

$\mathrm{q}_{\text {rad }}$ radiation heat $\mathrm{flux}, \mathrm{w} \cdot \mathrm{m}^{-2}$

$\mathrm{q}_{\mathrm{gas}}$ convection/conduction heat flux, $\mathrm{W} \cdot \mathrm{m}^{-2}$

$r$ radial coordinate, $m$

$\mathrm{R}$ universal gas constant, $\mathrm{J} \cdot \mathrm{mole}^{-1} \cdot \mathrm{K}^{-1}$
$\mathcal{R}^{S}$ heterogeneous reaction rate, mole $\cdot \mathrm{m}^{-2} \cdot \mathrm{s}^{-1}$

$\mathcal{R}_{\mathrm{k}}^{g}$ forward homogeneous reaction rate, mole $\cdot m^{-3} \cdot s^{-1}$

$\mathcal{R}_{-\mathrm{k}}^{g}$ reverse homogeneous reaction rate, $\mathrm{mole} \cdot \mathrm{m}^{-3} \cdot \mathrm{s}^{-1}$

$S$ number of surface reactions

$t$ time, $s$

$\mathrm{T}$ temperature, $\mathrm{K}$

$\mathrm{u}$ axial velocity, $\mathrm{m} \cdot \mathrm{s}^{-1}$

$v$ radial velocity, $\mathrm{m} \cdot \mathrm{s}^{-1}$

$z$ axial coordinate, $\mathrm{m}$

$\gamma$ surface-reaction probability

$\lambda$ thermal conductivity, $\mathrm{W} \cdot \mathrm{m}^{-1} \cdot \mathrm{K}^{-1}$

$\boldsymbol{\mu}$ dynamic viscosity, $\mathrm{kg} \cdot \mathrm{m}^{-1} \cdot \mathrm{s}^{-1}$

$v_{i k}$ stoichiometric coefficient for species $i$ in gas-phase reaction $k$

$\rho$ density, $\mathrm{kg} \cdot \mathrm{m}^{-3}$

$\sigma_{i k}$ stoichiometric coefficient for species $i$ in surface reaction $k$

$\omega \quad$ species mass fraction

\section{REFERENCES}

1. K.F. Jensen, Chem. Engng. Science, 42, 923 (1987)

2. D.W. Hess, K.F. Jensen and T.J. Anderson, Reviews in Chem. Eng., 3, 97 (1985)

3. M.L. Hitchman and B.J. Curtis, J. Crystal Growth, 60, 43 (1982)

4. H. Schlichting, "Boundary Layer Theory", McGraw-Hill, New York (1968)

5. G.H. Evans and R. Greif, Num. Heat Transfer, 14, 373 (1988)

6. R. Pollard and J. Newman, J. Electrochem. Soc., 127, 744 (1980)

7. M. Michaelidis and R. Pollard, J. Electrochem. Soc., 131, 860 (1984)

8. M.E. Coltrin, R.J. Kee and G.H. Evans, J. Electrochem. Soc., 136, 819 (1989)

9. M. Tirtowidjojo and R. Pollard, J. Crystal Growth, 93, 108 (1988)

10. G.H. Evans and R. Greif, J. Heat Transfer, 109, 928 (1987)

11. G.H. Evans and R. Greif, Num. Heat Transfer, 12, 243 (1987)

12. C. Houtman, D.B. Graves and K.F. Jensen, J. Electrochem. Soc., 133, 961 (1986)

13. P. Lee, D. McKenna, D. Kapur and K.F. Jensen, J. Cryst. Growth, 77, 120 (1986) 
14. H. Chehouani, B. Armas, S. Benet and S. Brunet, J. de Physique, Suppl. No.5, 50, C5-47 (1989)

15. M. Pons, R. Klein, C. Arena and S. Mariaux, J. de Physique, Suppl. No.5, 50, C5-47 (1989)

16. G. Wahl, Thin Solid Films, 40, 13 (1977)

17. D.I. Fotiadis, S. Kieda and K.F. Jensen, J. Cryst. Growth, 102, 441 (1990)

18. C. Weber, C. van Opdorp and M. de Keijser, J. Appl. Phys., 67, 2109 (1990)

19. R.B. Bird, W.E. Stewart and E.N. Lightfood, "Transport Phenomena", John Wiley \& Sons, New York (1960)

20. J.O. Hirschfelder, C.F. Curtiss and R.B. Bird, "Kinetic Theory of Gases and Liquids", John Wiley \& Sons, New York (1967)

21. R.C. Reid, J.M. Prausnitz and B.E. Poling, "The Properties of Gases and Liquids", McGraw-Hill, New York (1987)

22. C.R. Kleijn, T.H. van der Meer and C.J. Hoogendoorn, J. Electrochem. Soc., 136, 3423 (1989)

23. R.A. Svehla, NASA Technical Report R-132, 1962

24. For example: "Fluent-CVD" (Creare.x, Hanover, NH, USA), "Phoenics" (CHAM Ltd., Wimbledon, Gr. Britain, "FIDAP" (Fluid Dynamics Int., Evanston, IL. USA)

25. J.I. Ulacia F. and C. Werner, Solid State Techn., Nov. 1990, 107 (1990)

26. N.I. Buchan and J.M. Jasinski, J. Cryst Growth, 106, 227 (1990)

27. K.F. Roenigk, K.F. Jensen and R.W. Carr, J. Phys. Chem., 91, 5732 (1987)

28. M.E. Coltrin, R.J. Kee and J.A. Miller, J. Electrochem. Soc., 131, 425 (1984)

29. M.E. Coltrin, R.J. Kee and J.A. Miller, J. Electrochem. Soc., 133, 1206 (1986)

30. C.J. Giunta, J.D. Chapple-Sokol and R.G. Gordon, J. Electrochem. Soc., 137, 3237 (1990)

31. K.F. Jensen, T.J. Mountziaris and D.I. Fotiadis, in C.W. Tu, V.D. Mattera and A.C. Gossard (eds.), "III-V Heterostructures for Electronic/Photonic Devices", Materials Research Society, Pittsburgh (1989)

32. B.S. Meyerson and W. Olbricht, J. Electrochem. Soc., 131, 2361 (1984)

33. C. Vinante, P. Duverneuil and J.P. Couderc, J. de Physique, Suppl. No. 5, p. c5-35 (1989)

34. R. Arora and R. Pollard, in K.E. Spear and G.W. Cullen (eds.), Proc. 11th Int. Conf. on Chemical Vapor Deposition, p. 106, The Electrochemical Society, Pennington (1990)

35. R. Arora and R. Pollard, "A Mathematical Model for CVD Processes Influenced by Surface Reaction Kinetics: Application to Low Pressure Deposition of Tungsten", J. Electrochem. Soc., in print (1991)

36. N. Kobayashi, H. Goto and M. Suzuki, in K.E. Spear and G.W. Cullen (eds.), Proc. 11th Int. Conf. on Chemical Vapor Deposition, p. 434, The Electrochem. Society, Pennington (1990)

37. C.R. Kleijn, "A Mathematical Model of the Hydrodynamics and Gas-phase Reactions in Silicon LPCVD in a Single-Wafer Reactor", J. Electrochem. Soc., in print (1991)

38. A. Sherman, J. Electr. Mat., 17, 413 (1988)

39. R.J. Buss, P. Ho, W.G. Breiland and M.E. Coltrin, J. Appl. Phys., 63, 2808 (1988)

40. W.A.P. Claassen, J. Bloem, W.G.J.N. Valkenburg and C.H.J. van den Brekel, J. Crystal Growth, 57, 259 (1982)

41. C.M. McConica and K. Krishnamani, J. Electrochem. Soc., 133, 2542 (1986)

42. C.R. Kleijn and C.J. Hoogendoorn; A. Hasper, J. Holleman and J. Middelhoek, J. Electrochem. Soc., 138, 509 (1991)

43. M. Tirtowidjojo and R. Pollard, J. Crystal Growth, 98, 420 (1989)

44. J.I. Ulacia, S. Howell, H. Körner and Ch. Werner, Appl. Surf. Sci., 38, 370 (1989)

45. J.E.J. Schmitz, J.L.G. Suijker and M.J. Buiting, in R.S. Blewer and C.M. McConica (eds.), "Tungsten and Other Refractory Metals for VLSI Applications IV", p. 211, Materials Research Society, Pittsburgh (1989)

46. A. Hasper, personal communications, to be published

47. D.I. Fotiadis, M. Boekholt, K.F. Jensen and W. Richter, J. Cryst. Growth, 100, 577 (1990)

48. P.B. Chinoy, D.A. Kaminski and S.K. Ghandi, Num. Heat Transfer, Part A, 19, 85 (1991)

49. C.R. Kleijn and C.J. Hoogendoorn, Chem. Engng. Science, 46, 321 (1991)

50. E.J. McInerney, P. Geraghty and S. Kang, in S.S. Wong and S. Furukawa (eds.), "Tungsten and Other Advanced Metals for VLSI/ULSI Applications V", p. 135, Materials Researh Society, Pittsburgh (1990)

51. A. Hasper, J. Holleman, J. Middelhoek and C.R. Kleijn, in S.S. Wong and S. Furukawa (eds.), Tungsten and Other Advanced Metals for VLSI/ULSI Applications V, Materials Research Society, Pittsburgh, p. 127 (1990)

52. A. Hasper, J. Holleman and J. Middelhoek; C.R. Kleijn and C.J. Hoogendoorn, J. Electrochem. Soc., 138, in print (June 1991) 